\title{
Optical Properties of $U$ Centers in Alkali Halides and Alkaline Earth Fluorides
}

\author{
Herbert S. Bennett \\ Institute for Materials Research, National Bureau of Standards, Washington, D.C. 20234
}

(May 1, 1973)

\begin{abstract}
The two-electron orbitals for the $U$ center have been computed numerically from the HartreeFock-Slater (HFS) equations in the point-ion lattice potential. The lattice relaxation of the nearestneighbor ions is included in the model. The five lowest-lying $U$-center states for $\mathrm{NaCl}, \mathrm{KCl}, \mathrm{CdF}, \mathrm{CaF}_{2}$, $\mathrm{SrF}_{2}$, and $\mathrm{BaF}_{2}$ are given. The low-lying singlet states have the following order for increasing values of the energy: ${ }^{1} \mathrm{~S}(1 s, 1 s),{ }^{1} \mathrm{P}(1 s, 2 p)$, and ${ }^{1} \mathrm{~S}(1 s, 2 p)$. The energy levels for the triplet states ${ }^{3} \mathrm{~S}(1 s, 2 s)$, and ${ }^{3} \mathrm{P}(1 s, 2 p)$ lie between the energy levels for the ${ }^{1} \mathrm{~S}(1 s, 1 s)$ and ${ }^{1} \mathrm{P}(1 s, 2 p)$ states. The ordering of the triplet states depends upon the host crystal and the lattice relaxation. The predictions based upon the numerical HFS wave functions are compared with the predictions based upon past variational wave functions and with experiment.
\end{abstract}

Key words: $\mathrm{BaF}_{2} ; \mathrm{CaF}_{2}$; ionic polarization; $\mathrm{KCl}$; Mollwo-Ivey relations; $\mathrm{NaCl}$; point-ion potential; $\mathrm{SrF}_{2} ; U$ centers.

\section{Introduction}

The $U$ center in ionic crystals consists of a negative-hydrogen ion $\mathrm{H}^{-}$, located substitutionally at an anion site. Because the $\mathrm{H}^{-}$ion has a different mass, polarizability, and interaction with its neighboring ions than has the anion which it replaces, the $U$ center changes both the phonon spectrum and the optical properties of its host crystal. Changes in the phonon spectrum include new features at energies substantially greater than the energies of the optic phonons of the host lattice [1-11]. ${ }^{1}$ Changes in the optical properties include a characteristic electronic absorption band, the $U$ band, in the ultraviolet region [12-20] and are the concerns of this paper.

The author continues in this paper his calculations, reported in part in [21], on the several states of the $U$ center in the two alkali halides, $\mathrm{NaCl}$ and $\mathrm{KCl}$, and in the four alkaline earth fluorides, $\mathrm{CdF}_{2}, \mathrm{CaF}_{2}, \mathrm{SrF}_{2}$, and $\mathrm{BaF}_{2}$. Reference [21] contains discussions which stress the $U$ band transition energies between the singlet ground and first excited states. The author now reports his results on the spatial extent of the $U$-center

\footnotetext{
${ }^{1}$ Figures in brackets indicate the literature references at the end of this paper.
}

orbitals, on the transition energies between the first and second excited singlet states, and on the transition energies between the two lowest-lying truplet states. A point-ion lattice model is used which incorporates the Hartree-Fock-Slater (HFS) procedure to compute numerically the defect electron orbitals. The model includes estimates for the correlation energy of the singlet states [22], for the exchange energy of the triplet states [23], and for the lattice relaxation of the nearest-neighbor ions. The model also obeys completely the Franck-Condon principle that the lattice relaxation does not respond to rapid changes in the electronic state of the $U$ center when the $U$ center undergoes an optical transition. This means that all low-lying $U$-center states should have spatially compact electron orbitals, if the predictions of the model are to be internally consistent with its assumptions.

The present point-ion model has some limitations. Its numerical wave functions are not orthogonal to the electronic states of neighboring ions and thereby do not account properly for the finite size of the neighboring ions, particularly when the lattice relaxes. An extended-ion model which uses numerical wave functions instead of variational wave functions for the defect electron orbitals has not been reported. Such a calculation would require an amount of com- 
puter time which is substantially greater than that required by Wood and Öpik [18]. These authors solved extended-ion models by using trial wave functions with two variational parameters and by devising accurate approximations for efficiently computing Coulomb and exchange integrals. Their methods use Slater-type functions and are not applied readily to the present numerical procedures.

Keeping the above limitations in mind, we shall calculate here within the framework of the above model the energy levels of the three lowest-lying singlet states and the two lowest-lying triplet states. In some cases, these triplet states are degenerate or very nearly degenerate.

Because expressions for the change in energy of a classical point-ion lattice with lattice relaxation of the neighboring ions and expressions for the total energy of the $U$ center when the two electrons are in singlet and triplet states appear in sections 2 and 3 of [21], they are not repeated here. In section 2 of the present paper, the equations for absorption are given. Section 3 contains a discussion of the results and some remarks on the validity of point-ion models. Finally, the appendix contains a summary of the many assumptions upon which the HFS equations are based.

\section{Absorption}

Because no emission bands have been associated with the $U$ center [18], we shall study only the optical absorption which the defect electrons may undergo.

The initial state of an optical transition is a relaxed state, $\left|\eta_{i} ; \sigma_{i}\right\rangle$. The symbol $\eta$ denotes an electronic configuration of the two defect electrons which transforms as one of the following states, ${ }^{1} \mathrm{~S}(1 s, 1 s)$, ${ }^{3} \mathrm{~S}(1 s, 2 s),{ }^{3} \mathrm{P}(1 s, 2 p),{ }^{1} \mathrm{P}(1 s, 2 p)$, and ${ }^{1} \mathrm{~S}(1 s, 2 s)$. The quantity $\sigma$ characterizes the lattice relaxation; i.e., the $n$ nearest-neighbor ions of the $U$ center may move radially to the sites $\overrightarrow{\mathbf{r}}_{v}^{\prime}=\overrightarrow{\mathbf{r}}_{\nu}(1-\sigma)$ for $1 \leqslant \nu \leqslant n$. All other ions remain at their perfect lattice sites $\overrightarrow{\mathbf{r}}_{\nu}$. We minimize $E_{T}\left(\eta_{i} ; \sigma\right)$ with respect to the lattice relaxation $\sigma$ to obtain the energy of the initial state $E_{i}=E_{T}\left(\eta_{i} ; \sigma_{i}\right)$. The value $\sigma_{i}$ is that value of $\sigma$ for which $E_{T}\left(\eta_{i} ; \sigma_{i}\right)$ attains its minimum value. The $U$ center then undergoes an optical transition to an unrelaxed state $\mid \eta_{f}$; $\sigma_{i}>$, which has an electronic wave function calculated for the same distortion $\sigma_{i}$ as that for the initial state $\left|\eta_{i} ; \sigma_{i}\right\rangle$. The total energy of the final state is $E_{f}=E_{T}$ $\left(\eta_{f} ; \sigma_{i}\right)$. The optical absorption energy for a transition from state $i$ to state $f$ is

$$
E(i, f)=E_{T}\left(\eta_{f} ; \sigma_{i}\right)-E_{T}\left(\eta_{i} ; \sigma_{i}\right),
$$

where each term on the right-hand side of eq (1) is a negative number.

The expectation value of a given power of the radial coordinate $r$ gives us information on the spatial extent of the two-defect-electron wave functions. As a measure of the spatial extent, the author chooses to consider the first and third powers of $r$ for each of the orbitals; namely,

$$
r_{n l}\left[s, \eta\left(n l ; n^{\prime} l^{\prime}\right) ; \sigma\right]=r_{1}^{-s} \int R_{n l}(r) r^{s} R_{n l}(r) 4 \pi r^{2} d r
$$

and

$$
\begin{aligned}
& r_{n^{\prime} l^{\prime}}\left[s ; \eta\left(n l ; n^{\prime} l^{\prime}\right) ; \sigma\right] \\
& =r_{1}^{-s} \int R_{n^{\prime} l^{\prime}}(r) r^{s} R_{n^{\prime} l^{\prime}}(r) 4 \pi r^{2} d r,
\end{aligned}
$$

where $s=1$ or $s=3$ and $r_{1}$ is the nearest-neighbor distance of the perfect lattice. The principal and orbital quantum numbers for the radial wave functions $R_{n l}(r)$ are respectively $n$ and $l$. The ratio,

$$
r_{e}(n l)=\left[r_{n l}(s=3) / r_{n l}(s=1)\right],
$$

also indicates to what extent the radial functions have extended tails. The values of $r_{e}<1$ indicate compact states, and values of $r_{e}>1$ indicate diffuse states.

\section{Results and Conclusions}

In this section, the results of the point-ion model with lattice relaxation are reported. Table 1 contains the values of the input data which have been used.

TABLE 1. Input data for the point-ion model of the $U$ center with ionic polarization, exchange energy, and Coulomb-correlation energy. The Pauling factor of the $\nu$ th and $\mu$ th ions is $\beta_{\nu \mu}$. The ionic radius of the cation is $\rho_{+}$and the anion is $\rho_{-}$. The quantity $\rho$, having the units of length, is the stiffness factor in the empirical Born-Mayer exponential form which characterizes the repulsive energy between the $\nu$ th and $\mu$ th ions. The Madelung potential constant at the anion site is $\alpha_{M}$. The quantity $\bar{r}_{1}$ is the nearestneighbor distance (anion-cation) for the $\mathrm{NaCl}$ structure and is the lattice constant (cation-cation) for the $\mathrm{CaF}_{2}$ structure. The series coefficients $C_{4}, C_{6}$, and $C_{8}$ appear in the expansion in powers of the lattice distortion $\sigma$ for the change in electrostatic energy $E_{1}$ which occurs when a cation moves in the background of a perfect point-ion lattice potential; namely, $E_{1}=-\left(n / \bar{r}_{1}\right)\left(C_{4} \sigma^{4}+C_{6} \sigma^{6}\right.$ $\left.+C_{8} \sigma^{8}+\ldots\right)$, where $n$ is the number of nearest-neighbor cations to an anion. The quantities $\beta_{++^{\prime}} \beta_{+-}$and $\beta_{--^{\prime}}$ and $\alpha_{M}$ are dimensionless. All other quantities are expressed in terms of atomic units ( 1 a.u. of energy $=27.2 \mathrm{eV}=4.36$ attojoules and $1 \mathrm{a}$.u. of length $=0.0529 \mathrm{~nm}$ ).

\begin{tabular}{l|c|c|c|c|c|c}
\hline \hline & $\mathrm{NaCl}$ & $\mathrm{KCl}$ & $\mathrm{CdF}_{2}$ & $\mathrm{CaF}_{2}$ & $\mathrm{SrF}_{2}$ & $\mathrm{BaF}_{2}$ \\
\hline$\beta_{++}$ & 1.25 & 1.25 & 1.50 & 1.50 & 1.50 & 1.50 \\
$\beta_{+-}$ & 1.00 & 1.00 & 1.125 & 1.125 & 1.125 & 1.125 \\
$\beta_{--}$ & 0.75 & 0.75 & 0.75 & 0.75 & 0.75 & 0.75 \\
$\rho$ & $\mathrm{a} 0.599$ & $\mathrm{a} 0.637$ & 0.582 & $\mathrm{~b} 0.546$ & ${ }^{\mathrm{b}} 0.560$ & ${ }^{\mathrm{b}} 0.582$ \\
$\rho_{+}$ & $\mathrm{a} 2.21$ & $\mathrm{a} 2.77$ & 2.76 & ${ }^{\mathrm{c}} 2.21$ & ${ }^{\mathrm{c}} 2.48$ & ${ }^{\mathrm{c}} 2.76$ \\
$\rho_{-}$ & $\mathrm{a} 3.00$ & $\mathrm{a} 3.00$ & 1.98 & ${ }^{\mathrm{c}} 1.98$ & ${ }^{\mathrm{c}} 1.98$ & ${ }^{\mathrm{c}} 1.98$ \\
$\alpha_{M}$ & 1.748 & 1.748 & 4.071 & 4.071 & 4.071 & 4.071 \\
$\bar{r}_{1}$ & $\mathrm{a} 5.31$ & $\mathrm{a} 5.93$ & 10.21 & ${ }^{\mathrm{c}} 10.32$ & ${ }^{\mathrm{c}} 10.95$ & ${ }^{\mathrm{c}} 11.71$ \\
$C_{4}$ & $\mathrm{~d} 3.579$ & $\mathrm{~d} 3.579$ & $\mathrm{e} 1.865$ & $\mathrm{e} 1.865$ & ${ }^{\mathrm{e}} 1.865$ & ${ }^{\mathrm{e}} 1.865$ \\
$C_{6}$ & ${ }^{\mathrm{d}} 0.9895$ & $\mathrm{~d} 0.9895$ & & & & \\
$C_{8}$ & ${ }^{\mathrm{d}} 2.942$ & ${ }^{\mathrm{d}} 2.942$ & & & & \\
\hline
\end{tabular}

a M. P. Tosi, in Solid State Physics, edited by F. Seitz and D. Turnbull (Academic Press Inc., New York, 1964), Vol. XVI, p. 52.

${ }^{b}$ A. D. Franklin (private communication).

${ }^{c}$ G. C. Benson and E. Dempsey, Proc. Roy. Soc. (London) A266, 344 (1962).

d A. Scholz, Phys, Status Solidi 7, 973 (1964).

${ }^{\mathrm{e}}$ H. S. Bennett, J. Res. Nat. Bur. Stand. (U.S.), 72A (Phys. and Chem.), No. 5, 471-474 (Sept.-Oct. 1968). 
The numerical procedures give the energy eigenvalues to an accuracy of $|\Delta E| E \mid \approx 0.001$. They also give the self-consistent potential which appears in the HFS equations to an accuracy of $|\Delta V| V \mid \approx 0.01$, within the context of the point-ion model. The quantities $\Delta E$ and $\Delta V$ are, respectively, the changes in the trial eigenvalue and self-consistent potential which occur between two successive iterations in the numerical itegration procedure. The numerical details on the spatial extent of the five lowest-lying states of the $U$ center are reported only for $\mathrm{KCl}$ and $\mathrm{CaF}_{2}$ with no lattice relaxation. These two crystals are quantitatively representative of the remaining four crystals examined in this paper and illustrate respectively most of the salient features common to the NaCl-type and $\mathrm{CaF}_{2}$-type structures. Succeeding tables do contain, however, the energy levels for the five lowest-lying states in all six crystals. The computed data for the case in which the lattice relaxation and the electron orbitals are determined in a self-consistent manner are to within about 30 percent qualitatively similar to the computed data presented in tables 2 and 3 . The only exception to this is that the orbitals tend to be more compact when $\sigma>0$ and that the orbitals tend to be more diffuse when $\sigma<0$ than they are when $\sigma=0$ (no lattice relaxation). Tables 2 and 3 contain the numerical predictions of the point-ion model for $U$ centers in $\mathrm{KCl}$ and $\mathrm{CaF}_{2}$ when the lattice relaxation is zero $(\sigma=0)$. These two tables show that the ordering of the singlet energy levels with increasing energy is ${ }^{1} \mathrm{~S}(1 s, 1 s),{ }^{1} \mathrm{P}(1 s, 2 p)$, and ${ }^{1} \mathrm{~S}(1 s, 2 s)$. They also show that the energies of the triplet states ${ }^{3} \mathrm{~S}(1 s, 2 s)$ and ${ }^{3} \mathrm{P}(1 s$, $2 p$ ) lie between the ground singlet state energy and the first-excited-singlet state energy. The energies of these two low-lying triplet states lie close together and may be nearly degenerate as in the case for $\mathrm{KCl}$. In addition the ordering of these two triplet energy levels depends upon the crystal and upon the lattice relaxation. The results of [17] for the alkali halides predict the same ordering for the singlet energy levels. They also predict that the triplet ${ }^{3} \mathrm{~S}(1 s, 2 s)$ and ${ }^{3} \mathrm{P}(1 s, 2 p)$ energy levels lie between the ${ }^{1} \mathrm{~S}(1 s, 1 s)$ and ${ }^{1} \mathrm{P}(1 s, 2 p)$ energy levels. However, contrary to the present results, the results of [17] predict that the ${ }^{3} \mathrm{~S}(1 s, 2 s)$ energy level lies below and is well spearated from the ${ }^{3} \mathrm{P}(1 s$, $2 p$ ) energy level. These differences among the ordering of variational states and numerical triplet states may be explained by either or both of two possibilities: First, the authors of [17] have estimated the exchange terms in a different manner than the present author. And second, the numerical wave functions are more "flexible" than two-parameter-variational wave functions. One measure of the flexibility of a wave function is its second order-spatial derivative. The latter is proportional to the kinetic energy. Variational functions introduce artificial bounds on the kinetic energy and for some self-consistent potentials predict lower energy levels and different orderings of the states than numerical functions.

Table 4 gives for the six crystals the predicted energy levels, $U$-band transition energies, the $U$-band oscillator strengths, and the lattice relaxation of the nearest-neighbor ions. The oscillator strengths $f$ in
TABLE 2. The five lowest-lying states for $\mathrm{KCl}$ of the $U$ center in the perfect point-ion lattice $(\sigma=0)$ with exchange energy and Coulomb correlation energy. For convenience, the states ${ }^{1} \mathrm{~S}(1 s, 1 s),{ }^{3} \mathrm{P}(1 s$, $2 p),{ }^{3} \mathrm{~S}(1 s, 2 s),{ }^{1} \mathrm{P}(1 s, 2 p)$, and ${ }^{1} \mathrm{~S}(1 s, 2 s)$ are labeled $A, B, C, D$, and $E$, respectively, in this table. The total energy of state $X$ is $E_{T}(X$; $\sigma=0)$, where $X=A, B, C, D$, or $E$. The transition energy from state $X$ to state $Y$ is $E(X, Y)$. The quantities $n$ and $l$ are the principal and angular-momentum quantum numbers for the symmetry of the one-electron orbitals from which the $U$-center configuration is made. The spatial extent quantities $r_{n l}(s)$ and $r_{e}(n l)$ are dimensionless and the energies are expressed in terms of atomic units. (1 a.u. of energy $=27.2 \mathrm{eV}=4.36$ attojoules.)

\begin{tabular}{l|r|r|r|r|r}
\hline \multicolumn{1}{c|}{ State } & \multicolumn{1}{c|}{$A$} & \multicolumn{1}{c|}{$B$} & \multicolumn{1}{c|}{$C$} & \multicolumn{1}{c}{$D$} & \multicolumn{1}{c}{$E$} \\
\hline$E_{T}$ & -1.015 & -0.953 & -0.957 & -0.878 & -0.856 \\
$r_{n l}(1)$ & 0.565 & .281 & .285 & .479 & .531 \\
$r_{n l}(3)$ & .334 & .057 & .059 & .234 & .293 \\
$r_{e}(n l)$ & .590 & .202 & .208 & .490 & .551 \\
$r_{n^{\prime} l^{\prime}}(1)$ & .565 & .857 & .882 & .881 & .956 \\
$r_{n^{\prime} \prime^{\prime}}(3)$ & .334 & .868 & 1.005 & .929 & 1.915 \\
$r_{e}\left(n^{\prime} l^{\prime}\right)$ & .590 & 1.012 & 1.140 & 1.054 & 2.002 \\
& & & & & \\
$E(A, D)$ & .137 & & & & \\
$E(D, E)$ & .022 & & & & \\
$E(B, C)$ & .004 & & & & \\
\hline
\end{tabular}

TABLE 3. The five lowest-lying states for $\mathrm{CaF}_{2}$ of the $U$ center in the perfect point-ion lattice $(\sigma=0)$ with exchange energy and Coulomb correlation energy. The notation in this table is the same as the notation given in table 2 .

\begin{tabular}{l|r|r|r|r|r}
\hline \multicolumn{1}{c|}{ State } & \multicolumn{1}{c|}{$A$} & \multicolumn{1}{c|}{$B$} & \multicolumn{1}{c}{$C$} & \multicolumn{1}{c}{$D$} & \multicolumn{1}{c}{$E$} \\
\hline$E_{T}$ & -1.204 & -1.072 & -1.047 & -0.999 & -0.960 \\
$r_{n l}(1)$ & 0.626 & 0.374 & 0.372 & .559 & .553 \\
$r_{n l}(3)$ & .435 & .123 & .120 & .340 & .330 \\
$r_{e}(n l)$ & .694 & .328 & .323 & .607 & .597 \\
$r_{n^{\prime} l^{\prime}}(1)$ & .626 & .940 & 1.079 & .986 & 1.516 \\
$r_{n^{\prime} l^{\prime}}(3)$ & .435 & 1.268 & 2.180 & 1.455 & 1.608 \\
$r_{e}\left(n^{\prime} l^{\prime}\right)$ & .694 & 1.350 & 2.021 & 1.476 & 4.012 \\
& & & & & \\
$E(A, D)$ & .205 & & & & \\
$E(D, E)$ & .039 & & & & \\
$E(B, C)$ & .025 & & & & \\
\hline
\end{tabular}

TABLE 4. Energy levels and $U$-band transition energies for absorption from the ${ }^{1} \mathrm{~S}(1 s, 1 s)$ state to the ${ }^{1} \mathrm{P}(1 s, 2 p)$ state for the $U$ center. The lattice relaxation and Coulomb correlation are included. The initial state is ${ }^{1} \mathrm{~S}(1 s, 1 s)$ and $\sigma=\sigma_{i}$. The value of $\sigma$ remains the same for the final state ${ }^{1} \mathrm{P}(1 s, 2 p)$. For convenience, the states ${ }^{1} \mathrm{~S}(1 s, 1 s)$ and ${ }^{1} \mathrm{P}(1 s, 2 p)$ are labeled $A$ and $D$, respectively, in this table. The total energy of the state $X$ is $E_{T}(X ; \sigma)$, where $X=A$ or $D$. The $U$-band energy is $E(A, D)$. The experimental value for the $U$-band energy is $E(A, D$; expt). The theoretical oscillator strength is $f$. The quantities $\sigma_{i}$ and $f$ are dimensionless. All other quantities are in atomic units. (1 a.u. of energy $=27.2 \mathrm{eV}=4.36$ attojoules.)

\begin{tabular}{|c|c|c|c|c|c|c|}
\hline $\begin{array}{l}\text { Sub- } \\
\text { stance }\end{array}$ & $\sigma_{i}$ & $f$ & $E_{T}\left(A, \sigma_{i}\right)$ & $E_{T}\left(D ; \sigma_{i}\right)$ & $E(A, D)$ & $\begin{array}{c}E(A, D, \\
\quad \operatorname{expt})\end{array}$ \\
\hline $\mathrm{NaCl}$ & 0.041 & 1.90 & -1.087 & -0.914 & 0.173 & a 0.238 \\
\hline $\mathrm{KCl}$ & .060 & 1.90 & -1.023 & -.870 & .153 & a .213 \\
\hline $\mathrm{CdF}_{2}$ & .050 & 1.94 & -1.221 & -.987 & .234 & \\
\hline $\mathrm{CaF}_{2}$ & .060 & 1.94 & -1.221 & -.985 & .236 & b . 281 \\
\hline $\mathrm{SrF}_{2}$ & .057 & 1.94 & -1.177 & -.960 & .217 & b. 259 \\
\hline $\mathrm{BaF}_{2}$ & .065 & 1.94 & -1.138 & -.937 & .201 & b. .221 \\
\hline
\end{tabular}

a J. W. Schulman and W. D. Compton, Color Centers in Physics (The Macmillan Company, New York, 1962)

b J. H. Beaumont et al., J. of Physics C 3, L153 (1970). 
table 4 agree reasonably well with other theoretical calculations [1, 16,21].

Referring to tables 4 and 5 , we observe that including the lattice relaxation of the nearest-neighbor ions increases the predicted $U$-band transition energies. Nevertheless, the point-ion model with lattice relaxation still yields values for the $U$-band energies which are smaller than the observed values. Table 6 shows that these results are consistent with the other results predicted by point-ion models based upon variational wave functions.

One possible explanation for the energy levels of the singlet states in the present work lying above the corresponding energy levels in variational theories is given in [21].

Tables 7 and 8 give the energy levels and transition energies for absorption respectively from the ${ }^{1} \mathrm{P}(1 s$, $2 p)$ state to the ${ }^{1} \mathrm{~S}(1 s, 2 s)$ state and from the ${ }^{3} \mathrm{P}(1 s, 2 p)$ state to the ${ }^{3} \mathrm{~S}(1 s, 2 s)$ state.
TABLE 5. Energy levels and $U$-band transition energies for absorption from the ${ }^{1} S(1 s, 1 s)$ state to the ${ }^{1} P(1 s, 2 p)$ state for the $U$ center with no ionic polarization. Because $\sigma=0$, the lattice relaxation is zero. The Coulomb correlation is included. The initial state is ${ }^{1} S(1 s, 1 s)$ and $\sigma=\sigma_{1}=0$. For convenience, the states ${ }^{1} \mathrm{~S}(1 s, 1 s)$ and ${ }^{1} \mathrm{P}(1 s, 2 p)$ are labeled $A$ and $D$, respectively, in this table. The total energy of the state $X$ is $E_{T}(X ; \sigma)$, where $X=A$ or $D$. The $U$-band energy is $E(A, D)$. The experimental value for the $U$-band energy is $E\left(A, D\right.$; expt). The quantity $\sigma_{i}$ is dimensionless. All other quantities are in atomic units. (l a.u. of energy $=27.2 \mathrm{eV}=4.36$ attojoules.)

\begin{tabular}{l|r|r|r|r|r}
\hline \hline Substance & \multicolumn{1}{|c|}{$\sigma_{i}$} & $E_{T}\left(A ; \sigma_{i}\right)$ & $E_{T}\left(D ; \sigma_{i}\right)$ & $E(A, D)$ & $E(A, D ;$ expt $)$ \\
\hline $\mathrm{NaCl}$ & 0.0 & -1.082 & -0.921 & 0.161 & ${ }^{\mathrm{a}} 0.238$ \\
$\mathrm{KCl}$ & .0 & -1.015 & -.878 & .137 & ${ }^{\mathrm{a}} 0.213$ \\
$\mathrm{CdF}_{2}$ & .0 & -1.211 & -1.003 & .208 & \\
$\mathrm{CaF}_{2}$ & .0 & -1.204 & -0.999 & .205 & $\mathrm{~b} 0.281$ \\
$\mathrm{SrF}_{2}$ & .0 & -1.162 & -0.972 & .190 & b 0.259 \\
$\mathrm{BaF}_{2}$ & .0 & -1.117 & -0.943 & .174 & b 0.221 \\
\hline
\end{tabular}

a J. W. Schulman and W. D. Compton, Color Centers in Physics (The Macmillan Company, New York, 1962).

${ }^{b}$ J. H. Beaumont et al., J. of Physics C 3, L153 (1970).

TABLE 6. Comparisons among various theoretical values and experimental values for the U-band transition energies. This is the transition from the ${ }^{1} \mathrm{~S}(1 s, 1 s)$ state to the ${ }^{1} \mathrm{P}(1 s, 2 p)$ state. All energies are in atomic units (l a.u. of energy $=27.2 \mathrm{eV}=4.36$ attojoules).

\begin{tabular}{|c|c|c|c|c|c|c|c|}
\hline Substance & Experiment & Ref. a & Ref. b & Ref. c & Ref. d & Ref. e & $\begin{array}{c}\text { Present } \\
\text { work } \\
\text { (Table 4) }\end{array}$ \\
\hline $\begin{array}{l}\mathrm{NaCl} \\
\mathrm{KCl} \\
\mathrm{CdF}_{2} \\
\mathrm{CaF}_{2} \\
\mathrm{SrF}_{2} \\
\mathrm{BaF}_{2}\end{array}$ & $\begin{array}{r}\mathrm{f} \\
\mathrm{f} \\
\mathrm{f} .213 \\
\\
\mathrm{~g} .281 \\
\mathrm{~g} .259 \\
{ }^{\mathrm{g}} .221\end{array}$ & 0.205 & 0.219 & $\begin{array}{r}0.203 \\
.178\end{array}$ & $\begin{array}{r}0.202 \\
.178\end{array}$ & $\begin{array}{r}0.251 \\
.234 \\
.217\end{array}$ & $\begin{array}{r}0.173 \\
.153 \\
.234 \\
.236 \\
.217 \\
.201\end{array}$ \\
\hline
\end{tabular}

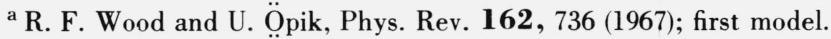

${ }^{b}$ R. F. Wood and U. Öpik, Phys. Rev. 162, 736 (1967); second model.

${ }^{c}$ H. N. Spector et al., J. Chem. Phys. 46, 2676 (1967).

${ }^{\text {d }}$ B. S. Gourary, Phys. Rev. 112, 337 (1958).

${ }^{e}$ R. S. Singh et al., J. Chem. Phys. 52, 2341 (1970).

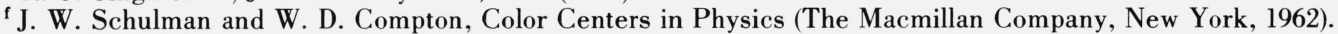

${ }^{g}$ J. H. Beaumont et al., J. of Physics C. 3, L153 (1970).

TABLE 7. Energy levels and second singlet transition energies for absorption from the ${ }^{1} \mathrm{P}(1 s, 2 p)$ state to the ${ }^{1} \mathrm{~S}(1 s, 2 s)$ state for the $U$ center. The lattice relaxation and Coulomb correlation are included. The initial state is ${ }^{1} \mathrm{P}(1 s, 2 p)$ and $\sigma=\sigma_{i}$. The value of $\sigma$ remains the same for the final state ${ }^{1} \mathrm{~S}(1 s, 2 s)$. For convenience, the states ${ }^{1} \mathrm{P}(1 s, 2 p)$ and ${ }^{1} \mathrm{~S}(1 s, 2 s)$ are labeled $D$ and $E$, respectively. The total energy of the state $X$ is $E_{T}(X ; \sigma)$, where $X=D$ or $E$. The second singlet transition energy is $E(D, E)$. The quantity $\sigma_{i}$ is dimensionless. All other quantities are in atomic units. ( 1 a.u. of energy $=27.2 \mathrm{eV}=4.36$ attojoules.)

\begin{tabular}{l|r|r|r|r}
\hline \hline Substance & \multicolumn{1}{|c|}{$\sigma_{i}$} & $E_{T}\left(D ; \sigma_{i}\right)$ & $E_{T}\left(E ; \sigma_{i}\right)$ & $E(D, E)$ \\
\hline $\mathrm{NaCl}$ & -0.011 & -0.921 & -0.894 & 0.028 \\
$\mathrm{KCl}$ & .000 & -.878 & -0.856 & 0.022 \\
$\mathrm{CdF}_{2}$ & -.082 & -1.010 & -1.010 & 0.000 \\
$\mathrm{CaF}_{2}$ & .000 & -0.999 & -0.960 & 0.039 \\
$\mathrm{SrF}_{2}$ & .001 & -.972 & -0.935 & 0.037 \\
$\mathrm{BaF}_{2}$ & .024 & .945 & -0.904 & 0.042 \\
\hline
\end{tabular}

TABLE 8. Energy levels and first triplet transition energies for transitions from the ${ }^{3} \mathrm{P}(1 s, 2 p)$ state to the ${ }^{3} \mathrm{~S}(1 s, 2 s)$ state for the $U$ center. The lattice relaxation and exchange energy are included. The initial state is ${ }^{3} \mathrm{P}(1 s, 2 p)$ and $\sigma=\sigma_{i}$. The value of $\sigma$ remains the same for the final state ${ }^{3} \mathrm{~S}(1 s, 2 s)$. These states are labeled $B$ and $C$, respectively, for convenience. The total energy of the state $X$ is $E_{T}(X, \sigma)$, where $X=B$ or $C$. The first triplet transition energy is $E(B, C)$. The quantity $\sigma_{i}$ is dimensionless. All other quantities are in atomic units. (1 a.u. of energy $=27.2$ $\mathrm{eV}=4.36$ attojoules.)

\begin{tabular}{l|r|r|r|r}
\hline \hline Substance & \multicolumn{1}{|c|}{$\sigma_{i}$} & $E_{T}\left(B ; \sigma_{i}\right)$ & $E_{T}\left(C ; \sigma_{i}\right)$ & $E(B, C)$ \\
\hline $\mathrm{NaCl}$ & -0.006 & -0.996 & -0.992 & 0.004 \\
$\mathrm{KCl}$ & 0.004 & -0.953 & -0.957 & -0.004 \\
$\mathrm{CdF}_{2}$ & -0.014 & -1.077 & -1.057 & 0.021 \\
$\mathrm{CaF}_{2}$ & 0.009 & -1.072 & -1.044 & 0.029 \\
$\mathrm{SrF}_{2}$ & 0.006 & -1.046 & -1.026 & 0.020 \\
$\mathrm{BaF}_{2}$ & 0.028 & -1.020 & -1.004 & 0.017 \\
\hline
\end{tabular}


A Mollwo-Ivey relation [24].

$$
E=\alpha a^{-n},
$$

with $n \approx 1.1$ relates the observed energies $E$ at the peak of the $U$-band in many alkali halides to the lattice spacing $a$. However, the observed energies of the $U$-band peaks in the alkaline earth fluorides do not fit a Mollwo-Ivey empirically-derived ralation [20]. A similar situation occurs for the $F$-band peaks. A Mollwo-Ivey relation with $n \approx 1.84$ predicts quite well the locations of the $F$-band peaks in many NaCltype alkali halides. But, such a relation does not predict the observed $F$-band peaks in the alkaline earth fluorides [20].

Point-ion models for the $U$-band which do not include lattice relaxation yield $U$-band energies which follow Mollwo-Ivey relations. The variational-point-ion theory of [17] predicts $U$-band energies for $\mathrm{NaCl}$ type crystals which obey a Mollwo-Ivey relation with $n \approx 1.14$. The similar variational theory of [1] gives $U$-band energies for the alkaline earth fluorides which follow reasonably well a Mollwo-Ivey relation with $\mathrm{n} \approx 1.143$. The present point-ion model in which the lattice relaxation and the coulomb correlation term $W(r)$ are zero predicts that the Mollwo-Ivey exponents are 1.22 for $U$-bands in $\mathrm{NaCl}$ and $\mathrm{KCl}$ and 1.31 for $U$ bands in the fluorides $\mathrm{CdF}_{2}, \mathrm{CaF}_{2}, \mathrm{SrF}_{2}$, and $\mathrm{BaF}_{2}$. When the Coulomb correlation term $W(r)$ in a pointion model with no lattice relaxation is included, the Mollwo-Ivey exponents become, respectively, 1.46 and 1.33. The predicted Mollwo-Ivey exponents for the alkali halides which are obtained by variational methods are closer to the experimental values than those exponents which are obtained by the present numerical methods. However, when the lattice relaxation is included, then the predicted $U$-band energies for both the alkali halides and the fluorides do not follow Mollwo-Ivey relations.

The above results show that solving the HFS equations for the point-ion model of the $U$ center by numerical techniques leads to a greater disagreement between theory and experiment for the $U$-band energies than does solving the point-ion model by variational techniques. Also, the Mollwo-Ivey exponent obtained from the numerical HFS wave functions differs more from the observed exponent for $U$-bands in the alkali halides than does the exponent obtained from the variational wave functions. These greater disagreements between the predictions of the numerical HFS wave functions and predictions of the variational wave functions for the point-ion model suggest that the past successes of the point-ion model have been fortuitous. More generally, the disagreement among the observed and computed values reported in this paper lead one to conclude that the point-ion models do not represent satisfactorily the properties of the $U$-center. The present work also emphasizes a need for models containing more detailed treatment of ionic polarization, electronic polarization, and the finite extent of the neighboring ions. Examples of two such models for the alkali halides appear in [18].
Whenever both of the $U$-center electrons have compact wave functions, the $U$ center (proton, anion vacancy and two electrons) is essentially neutral. In this case, it would produce only small, long-range polarizations, if any. The existence of these conditions then would justify treating all ions as point charges. Hence, the point-ion model is a reasonable approximation for the case in which most of the charge associated with the $U$-center electrons is localized about the proton-anion vacancy site and remains outside the neighboring ionic cores. The defect electrons then would experience a potential which scales with the lattice constant and the predicted $U$ bands would vary with the lattice constant, to a good approximation, according to a Mollow-Ivey relation.

The observed $U$ bands in the alkali halides satisfy a Mollwo-Ivey relation; those in the alkaline earth fluorides do not. The failure of the observed $U$ bands in the alkaline earth fluorides to obey a Mollwo-Ivey relation suggests the speculation that extended ion effects are more important in the alkaline earth fluorides than they are in the alkali halides. Extended ion effects include exchange and overlap among the $U$-center electrons and the core electrons of neighboring ions. These effects probably do not depend smoothly upon the lattice constant. It is interesting to notice in table 6 that most of the predicted $U$ bands in the alkaline earth fluorides are closer to the observed values than the predicted $U$ bands in the alkali halides. Nevertheless, using a point-ion model to describe the $U$ center in the alkaline earth fluorides is more questionable than using a point-ion model to describe the $U$ center in the alkali halides because the former class of $U$ centers fails to follow a Mollwo-Ivey relation.

The author thanks A. D. Franklin for many helpful discussions. He also thanks the personnel of the Aspen Center for Physics, where portions of the manuscript were written, for their kind hospitality.

\section{Appendix. Assumptions Contained in the HFS Equations}

Because the Schrödinger equation for the two-electron $U$ center,

$$
H_{T}(\mathbf{x}, \mathbf{y} ; \sigma) \Psi_{\eta}(x, y)=E \Psi_{\eta}(x, y),
$$

cannot be solved exactly, we use the self-consistent field method [Hartree-Fock (HF)] to calculate the wave functions of the $U$ center. The wave functions for the self-consistent field approximation are antisymmetrized sums of products of one-electron functions $u_{1}(r)$ and $u_{2}(r)$ :

$$
\begin{aligned}
\Psi_{\eta}(x, y) \approx \Psi_{\eta}(x, y ; H F) & \\
& =2^{-1 / 2}\left[u_{1}(x) u_{2}(y)-u_{1}(y) u_{2}(x)\right] .
\end{aligned}
$$


The functions $u$ have a restricted-central-field representation given by

$$
u_{n l m s}=R_{n l}(r) y_{l m}(\theta, \phi) \alpha_{s},
$$

where $R_{n l}(r)$ is the radial function, $Y_{l m}$ is a spherical harmonic function, and $\alpha_{s}$ is the spin function. The principal, orbital, magnetic, and spin quantum numbers are, respectively, $n, l, m$, and $s$. The radial functions $P_{n l}(r), P_{n l}(r)=r R_{n l}(r)$, are normalized to the crystal volume $\int P_{n l}{ }^{2}(r) d r=1$. The spherically averaged total electronic charge density for both spins in terms of the radial functions becomes

$$
\rho_{\mathrm{av}}(r)=-e\left[\sigma(r) / 4 \pi r^{2}\right],
$$

where the spherical density $\sigma(r)$ is

$$
\sigma(r)=\sum_{n l} \omega_{n l} P_{n l}^{2}(r),
$$

and the occupation number of the spatial orbital for both spins is $\omega_{n l}$. The summation $\Sigma_{n l} \omega_{n l}$ equals 2 for the $U$ center.

In terms of the above assumptions, the Coulomb potential $V_{c}(r)$ for the HFS equations then has the form:

$$
V_{c}(r)=\frac{e^{2}}{r} \int_{0}^{r} \sigma(t) d t+e^{2} \int_{r}^{\infty} \frac{\sigma(t)}{t} d t .
$$

Slater [23] suggests that the essential features of the HF method are retained when a common exchange potential replaces the exchange potentials for each of the orbitals $u_{1}$ and $u_{2}$. Applying his suggestion to the triplet states of the $U$ center, we assume that the common exchange potential $V_{e}(r)$ for the $U$ center at the point $r$ is equal to the exchange potential which a free-electron gas would have if its total electron charge density for both spins were equal to that of the nonuniform system (the two $U$-center defect electrons): namely,

$$
V_{e}(r)=-3 e^{2}\left[(3 / 8 \pi) e^{-1}\left|\rho_{a v}(r)\right|\right]^{1 / 3} .
$$

Let us define $V_{s}(r)=V_{c}(r)+V_{e}(r)$ for future use. The self-Coulomb energy part of the total electronic Coulomb energy cancels exactly in the conventional HF method a corresponding energy in the total exchange energy. This cancellation does not occur in the HFS equations at large $r$. We alter the sum $V_{s}(r)$ so that it has the correct asymptotic behavior at large $r$. We define $V(r)=V_{c}(r)+V_{e}(r)$ for $r<r_{0}$ and $V(r)$ $=e^{2}\left(\Sigma_{n l} \omega_{n l}-1\right) / r$ for $r \geqslant r_{0}$. The radius $r_{0}$ is that value of $r$ at which

$$
V_{s}\left(r_{0}\right)=e^{2}\left(\sum_{n l} \omega_{n l}=1\right) / r_{0} .
$$

The HF method also does not include the spatial correlation in the motion of the two defect electrons produced by their instantaneous Coulomb repulsion
$H_{c}(\mathbf{x}, \mathbf{x})$. The approximate HF wave function is the source of this Coulomb correlation problem because it does not depend upon the distance between the two electrons $|\mathbf{x}-\mathbf{y}|$. We define the correlation energy by the relation

$$
\begin{aligned}
E_{c}=\left\langle\Psi_{\eta}(x, y)\right. & \left.\left|H_{T}\right| \Psi_{\eta}(x, y)\right\rangle \\
& -\left\langle\Psi^{\eta}(x, y ; H F)\left|H_{T}\right| \Psi_{\eta}(x, y ; H F)\right\rangle .
\end{aligned}
$$

Let us assume also that a correlation operator $\epsilon_{c}(\mid \mathbf{x}$ $-\mathbf{y} \mid)$ exists such that its expectation value in the approximate representation $\Psi_{\eta}(\mathbf{x}, \mathbf{y} ; H F)$ is the correlation energy

$$
\boldsymbol{E}_{c}=\left\langle\Psi_{\eta}\left(x, y ; H F\left|\boldsymbol{\epsilon}_{c}(|\mathbf{x}-\mathbf{y}|)\right| \Psi_{\eta}(x, y ; H F)\right\rangle .\right.
$$

We may introduce, then, the correlation potential $W_{c}(r)$ by the following operation:

$$
W_{c}(r)=\int d^{3} x \Psi_{\eta}(x, r ; H F) \epsilon_{c}(|\mathbf{x}-\mathbf{r}|) \Psi_{\eta}(x, r ; H F) .
$$

Mitler [22] adapts to atomic helium the free-electron gas approximation for the correlation energy. He introduces in the $\mathrm{HF}$ equations an additional central "correlation" potential $W(r)$ to which pairs of electrons with opposite spin are subject. The approximate correlation potential $W(r)$ has the form [22]

$$
W(r)=-e^{2} \frac{0.288}{5.1 a_{0}+r_{s}(r)},
$$

where $a_{0}$ is the Bohr radius and the local density of electrons is

$$
e^{-1}|\rho(r)|=\left[(4 \pi / 3) r^{2}(r)\right]^{-1} .
$$

We expect that the expression for $W(r)$ is a good estimate for the correlation potential $W_{c}(r)$. Mitler [22] applies the expression for $W(r)$ to the ground state for helium and obtaines by perturbation theory a ground state energy which agrees to within $\frac{1}{2}$ percent of the experimental value. Our prescription is, then, to replace $V_{c}(r)$ in the singlet HFS variational equations with $V_{c}(r)+W(r)$. Because the inequality

$0 \leqslant W\left(r_{s}\right) / V_{\text {exch }}\left(r_{s}\right)$

$$
\leqslant 0.314 r_{s} /\left(5.1 a_{0}+r_{s}\right) \leqslant 0.314 r_{s}
$$

is obtained, we do not include the Coulomb correlation potential in the triplet HFS variational equations.

\section{References}

[1] Singh, R. S., et al., J. Chem. Phys. 52, 4341 (1970).

[2] Dötsch, H., and Mitra, S. S., Phys. Rev. 178, 1492 (1969).

[3] Schulman, J. H., and Compton, W. Dale, Color Centers in Solids (The Macmillan Company, New York, 1962).

[4] Klein, M. V., in Physics of Color Centers, edited by W. Beall Fowler (Academic Press, New York, 1968).

[5] Bilz, H., et al., J. Phys. Suppl. 27, C2 (1966). 
[6] Mitra, S. S., and Singh, R. S., Phys. Rev. Letters 16, 694 (1966).

[7] Wood, R. F., and Gilbert, R. L., Phys. Rev. 162, 746 (1967).

[8] Hayes W., and Mac Donald, H. F., Proc. Roy Soc. (London) A297, 503 (1967).

[9] Elliot, R. J., et al., Proc. Roy. Soc. (London) A289, 1 (1966).

10] Harrington, J. A., et al., (unpublished).

[11] Hall, J. L., and Schumacher, R. T., Phys. Rev. 127, 1892 (1962).

[12] Hilsch, R., and Pohl, R., Trans. Faraday Soc. 34, 883 (1938).

[13] Martienssen, W., Z. Physik 131, 488 (1952).

[14] Baldini, G., et al., Phys. Rev. 140, A2094 (1965).
[15] Mitra, S. S., and Brada, Y., Phys. Rev. 145, 626 (1966).

[16] Gourary, B. S., Phys. Rev. 112, 337 (1958).

[17] Spector, H. N., et al., J. Chem. Phys. 46, 2676 (1967).

[18] Wood, R. F., and Öpik, U., Phys. Rev. 162, 736 (1967).

[19] Sennett, C. T., J. Phys. Chem. Solids 26, 1097 (1965).

[20] Beaumont, J. H., et al., J. Phys. C 3, 152 (L) (1970).

[21] Bennett, H. S., Phys. Rev. B 6, 3936 (1972).

[22] Mitler, H., Phys. Rev. 99, 1835 (1955).

23] Slater, J. C., Phys. Rev. 81, 385 (1951).

[24] Ivey, R. F., Phys. Rev. 72, 341 (1947).

(Paper 77A5-788) 